\title{
Three-Dimensional Morphology of the Left Ventricular Outflow Tract: Impact on Grading Aortic Stenosis Severity
}

\author{
Luis Caballero, MD, PhD, Daniel Saura, MD, PhD, María José Oliva-Sandoval, MD, PhD, \\ Josefa González-Carrillo, MD, PhD, María Dolores Espinosa, MD, Miguel García-Navarro, MD, PhD, \\ Mariano Valdés, MD, PhD, Patrizio Lancellotti, MD, PhD, and Gonzalo de la Morena, MD, PhD, Murcia, Spain; \\ Liège, Belgium; and Lugo, Italy
}

Background: Left ventricular outflow tract (LVOT) measurement is a critical step in the quantification of aortic valve area. The assumption of a circular morphology of the LVOT may induce some errors. The aim of this study was to assess the three-dimensional (3D) morphology of the LVOT and its impact on grading aortic stenosis severity.

Methods: Fifty-eight patients with aortic stenosis were studied retrospectively. LVOT dimensions were measured using 3D transesophageal echocardiography at three levels: at the hinge points (HP) of the aortic valve and at 4 and $8 \mathrm{~mm}$ proximal to the annular plane. Results were compared with standard twodimensional echocardiographic measurements.

Results: Three-dimensional transesophageal echocardiography showed a funnel shape that was more circular at the HP and more elliptical at 4 and $8 \mathrm{~mm}$ proximal to the annular plane (circularity index $=0.92$ vs 0.83 vs 0.76 , $P<$.001). Cross-sectional area was smaller at the HP and larger at 4 and $8 \mathrm{~mm}$ from the annular plane (3.6 vs 3.9 vs $\left.4.1 \mathrm{~cm}^{2}, P=.001\right)$. The best correlation between two-dimensional and 3D transesophageal echocardiographic dimensions was at the HP (intraclass correlation coefficient $=0.75 ; 95 \% \mathrm{Cl}, 0.59-0.86$ ). When the HP approach was selected, there was a reduction in the percentage of patients with low flow (from $41 \%$ to $29 \%$ ).

Conclusions: A large portion of patients with aortic stenosis have funnel-shaped and elliptical LVOTs, a morphology that is more pronounced in the region farther from the annular plane. Two-dimensional LVOT measurement closer to the annular plane has the best correlation with 3D measurements. Measurement of the LVOT closer to the annular plane should be encouraged to reduce measurement errors. (J Am Soc Echocardiogr 2017;30:28-35.)

Keywords: Left ventricular outflow tract, Aortic stenosis, 3D echocardiography

Echocardiography is the standard imaging modality for the evaluation and management of patients with aortic stenosis (AS).$^{1-3}$ Effective aortic valve area (AVA) and transvalvular gradients are the principal measures for grading stenosis severity. Left ventricular outflow tract (LVOT) measurement is a critical step in the quantification of AVA by the continuity equation. Current recommendations on

From Servicio de Cardiología, IMIB-Arrixaca-UMU, Hospital Clínico Universitario Virgen de la Arrixaca, Murcia, Spain (L.C., D.S., M.J.O.-S., J.G.-C., M.D.E., M.G.-N., M.V., G.d.I.M.); GIGA-Cardiovascular Sciences, Department of Cardiology, University of Liège, Liège, Belgium (P.L.); and Gruppo Villa Maria Care and Research, E.S. Health Science Foundation, Lugo, Italy (P.L.).

Reprint requests: Gonzalo de la Morena, MD, PhD, Servicio de Cardiología, Hospital Clínico Universitario Virgen de la Arrixaca, Carretera Murcia Cartagena SN, 30120 Murcia, Spain (E-mail: gdlmorena@yahoo.es).

0894-7317/ $\$ 36.00$

Copyright 2016 by the American Society of Echocardiography.

http://dx.doi.org/10.1016/j.echo.2016.10.006 echocardiography advise measuring the LVOT on the longitudinal long-axis plane, at mid-systole and at 4 to $8 \mathrm{~mm}$ proximal to the annular plane. ${ }^{4}$ More recently, some other investigators have suggested $2 \mathrm{~mm}$ as the recommended point for measuring the LVOT. $^{5,6}$ Furthermore, the main limitation pointed out for this method is that the continuity equation assumes a circular morphology of the LVOT, whereas several studies have demonstrated its elliptical shape, and the frequent underestimation of AVA with this assumption compared with planimetered area. ${ }^{5-9}$ As a result, there are currently some concerns and controversies regarding the most appropriate method to measure the LVOT.

Three-dimensional (3D) transesophageal echocardiography (TEE) allows real-time volume rendering of images and offline volumetric quantification techniques. ${ }^{10}$ This method allows exploration of the morphology of the LVOT and measurement of its dimensions at any distance from the annular plane, without making geometric assumptions. Previous studies using this method confirmed the elliptical shape of the LVOT, but all of them were limited to the analysis of a 


\section{Abbreviations}

AS $=$ Aortic stenosis

AVA $=$ Aortic valve area

CSA $=$ Cross-sectional area

$\mathbf{H P}=$ Hinge points

ICC $=$ Intraclass correlation coefficient

LVOT $=$ Left ventricular outflow tract

MDCT $=$ Multidetector computed tomography

$\mathbf{S V i}=$ Stroke volume index

TEE $=$ Transesophageal echocardiography

3D = Three-dimensional

TTE $=$ Transthoracic echocardiography

VTI = Velocity-time integra single plane next to the insertion of the aortic valves. $5,6,9,11$ Nevertheless, the LVOT is a more complex structure that extends from the annulus of the aortic valve to 8 or $12 \mathrm{~mm}$ into the left ventricle. The present study was conducted to assess the morphology and size of the LVOT using 3D TEE along its $8 \mathrm{~mm}$ proximal to the annular plane and its impact on AVA estimated by the continuity equation.

\section{METHODS}

A retrospective study was conducted, including 58 patients from a series of 62 consecutive patients referred to our echocardiography laboratory for preoperative evaluation between January 2010 and May 2012. Transthoracic echocardiography (TTE) and 3D TEE were performed as part of the protocol for patients who were candidates for transcatheter aortic valve implantation. Two-dimensional TTE and 3D TEE were performed with a time interval of $<20$ days between them. The local clinical research ethics committee approved the protocol, and informed consent was obtained for all patients. In case of poor image quality, patients were excluded from the analysis.

\section{D TTE}

Two-dimensional TTE was performed using an iE33 ultrasound system equipped with an S5-1 phased-array transducer (Philips Medical Systems, Andover, MA). A systematic imaging protocol was performed by European Association of Cardiovascular Imagingaccredited cardiologists closely following current guidelines. ${ }^{1,4,12}$ The LVOT was measured by two independent observers on a zoomed longitudinal 2D image, in a mid-systolic frame, using the inner edge-to-inner edge technique and at the hinge points (HP) of the aortic valve. Referent LVOT measurement was performed $4 \mathrm{~mm}$ and $8 \mathrm{~mm}$ proximal to the annulus, in accordance with guideline recommendations. ${ }^{2,4}$ Each observer was free to choose any point as long as it was within 4 to $8 \mathrm{~mm}$ set in the LVOT. Doppler flow data were acquired from the LVOT, 2 to $4 \mathrm{~mm}$ below the annulus, in pulsedwave mode from the apical five-chamber view. The position of the sample volume was moved distally in the LVOT, toward the aortic valve, until a much steeper rise was obtained, as recommended. ${ }^{13}$ Pulsed Doppler spectral strips of LVOT systolic flow were manually traced on the modal curve. Aortic valve flows were obtained from all accessible acoustic positions, including the right parasternal window, to ensure maximal transaortic velocity measurement. The effec tive AVA was estimated using the continuity equation, averaging measurements from three different cardiac cycles (10 cardiac cycles if the patient was in atrial fibrillation) and assuming a circular shape of the LVOT. Image processing was performed offline using an image management system (Xcelera; Philips Medical Systems).

\section{D TEE}

Three-dimensional TEE was performed using the iE33 ultrasound system equipped with a matrix-array $\mathrm{X} 7-2 \mathrm{t}$ transducer (Philips Medical Systems). Sedation with intravenous propofol $(1 \mathrm{mg} / \mathrm{kg}$ followed by $0.5 \mathrm{mg} / \mathrm{kg}$ every 3-5 min) was used to minimize distress. Real-time $3 \mathrm{D}$ imaging of a pyramidal $60^{\circ} \times 30^{\circ}$ data set of the aortic valve and LVOT was obtained. Settings were optimized using narrowangled acquisition mode to ensure a volume rate up to $25 \mathrm{~Hz}{ }^{14}$ QLAB (3DQ module) (Philips Medical Systems) was used for postprocessing of images. In a mid-systolic frame (defined as maximal aortic valve opening on 3D transesophageal volume-rendered images), two orthogonal planes with their lines parallel to the longitudinal edge were set through the LVOT. A third plane was traced perpendicular to the previous two planes. This plane was reoriented as needed to ensure the most circular orifice area of the slot. Using the multislice tool of the software, we drew different cuts of the LVOT. First, we selected the minimum number of planes for the software tool, four planes. We adjusted the first plane to coincide with the HP of the aortic sigmoid cusps. Next, we adjusted the distance between the different planes to $4 \mathrm{~mm}$. In this way, we obtained four cuts of the LVOT: at the HP of the aortic leaflets, at $4 \mathrm{~mm}$ proximal to the annular plane, and also at $8 \mathrm{~mm}$ proximal to the annular plane. The fourth plane, the farthest from the aortic valve, usually passes through the proximal part of the anterior mitral leaflet, so it was not considered for LVOT analysis (Figure 1). A subsequent selection of the appropriate picture allowed us to measure the diameters (anteroposterior and medial-lateral) and cross-sectional area (CSA) by planimetry of the LVOT (Figure 2). LVOT morphology was evaluated by means of the circularity index dividing anteroposterior (short) by medial-lateral (large) diameters. A circularity index of 1 would represent a perfect circle, while a progressively lower value would represent a more ellipsoid geometry. The 3D assessment of AVA was made using the continuity equation and combining the Doppler velocity-time integral (VTI), measured in the LVOT, with the LVOT CSA determined at the three levels.

Data were reanalyzed in the whole cohort of patients with the same images and videos, and 2D transthoracic echocardiographic LVOT dimensions were remeasured by the same cardiologist and by a second observer in a blinded fashion, with a time interval of 2 to 7 days. A different observer blinded to the results of 2D TTE performed 3D image processing offline.

\section{Statistical Analysis}

Normal distribution of continuous variables was assessed using the Kolmogorov-Smirnov test (Lilliefors correction). Variable magnitudes are described as proportions, mean $\pm \mathrm{SD}$, or median (interquartile range) as appropriate. Differences between variables were assessed using the paired $t$ test, analysis of variance, or the Mann-Whitney $U$ test as appropriate. Differences between proportions were assessed using the $\chi^{2}$ test. Agreement between observers and imaging modalities was assessed using the intraclass correlation coefficient (ICC) Two-tailed $P$ values $<.05$ were considered to indicate statistical significance. Analysis was performed using SPSS version 15.0 (SPSS Chicago, IL)

\section{RESULTS}

Four patients were excluded from the analysis because of inadequate images. The demographic and echocardiographic characteristics of the 58 patients included are depicted in Table 1 . AVA was $<1 \mathrm{~cm}^{2}$ 


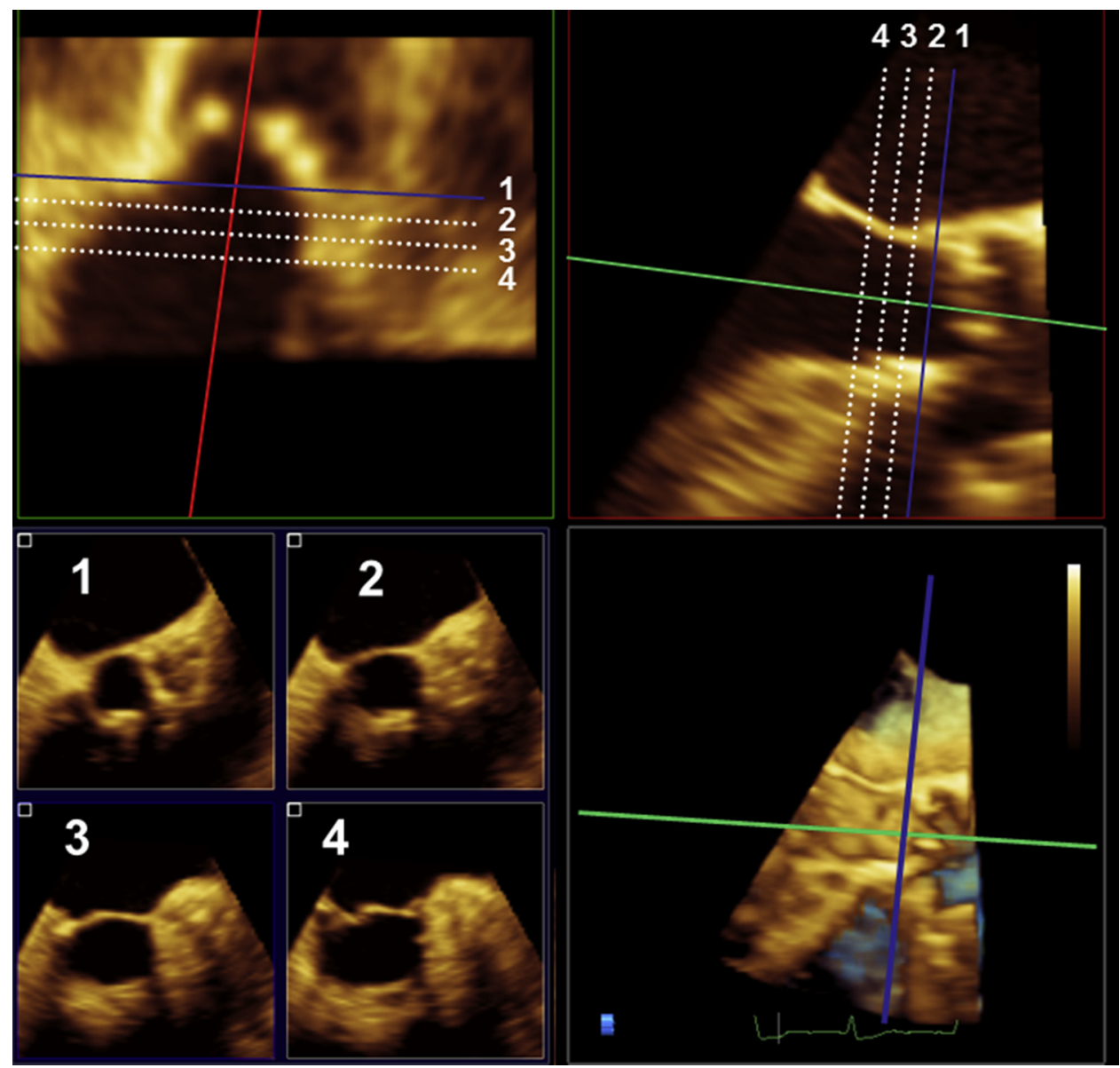

Figure 1 LVOT assessed by 3D TEE and multiplanar reconstruction with QLAB and multislice tool. (Top) The two images represent two orthogonal planes with their lines parallel to the longitudinal edge set through the LVOT. (Bottom) The left image corresponds to a third plane traced perpendicular to the previous planes to obtain the CSA of LVOT. Each line (1-4) corresponds to the four planes traced by QLAB multislice tool, with $4 \mathrm{~mm}$ between each. For measurements, we selected slice 1 at the HP, slice 2 at $4 \mathrm{~mm}$, and slice 3 at $8 \mathrm{~mm}$ into the LVOT.
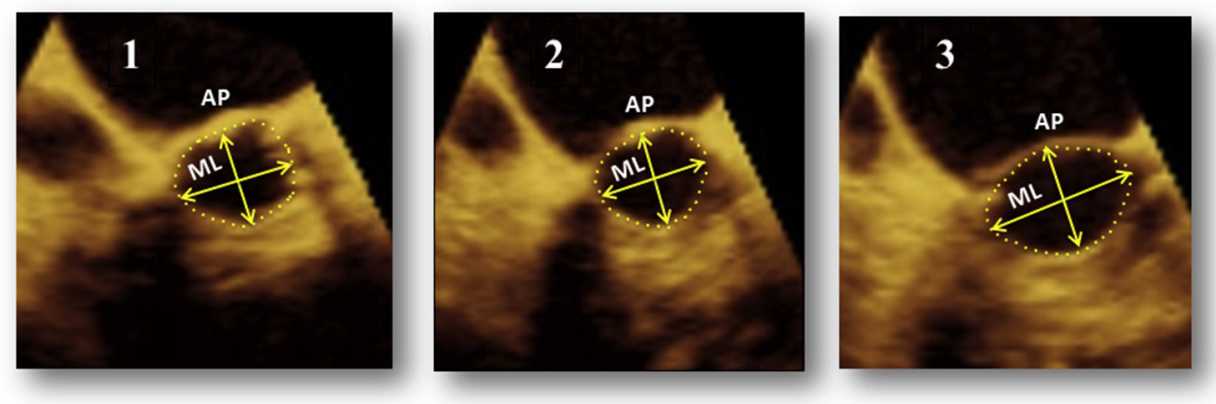

Figure 2 LVOT measurements at the HP of the aortic valve (1), at $4 \mathrm{~mm}$ (2), and at $8 \mathrm{~mm}$ (3) proximal to the aortic annulus. These slices correspond to those in Figure 1 and show how to measure the anteroposterior (AP) diameter, medial-lateral (ML) diameter, and CSA of the LVOT (dotted lines).

in 50 patients and between 1 and $1.3 \mathrm{~cm}^{2}$ in the remaining eight patients.

\section{Three-Dimensional Transesophageal Echocardiographic Morphology of the LVOT}

LVOT morphology assessed by 3D TEE showed a funnel shape (Figure 1). LVOT CSA was smaller at the level of the HP of the aortic valve and larger when measured at 4 and $8 \mathrm{~mm}$ proximal to the annular plane $\left(3.76 \pm 0.9\right.$ vs $4.05 \pm 1.1$ vs $4.46 \pm 1.3 \mathrm{~cm}^{2}$, $P<.001)$. Only 11 patients $(18.9 \%)$ had CSAs of the LVOT that were larger at the HP of the aortic valve than measured at $8 \mathrm{~mm}$ proximal to the annular plane. These patients had increases of interventricular septal thickness $(16.2 \pm 4.2$ vs $14.2 \pm 2.2 \mathrm{~mm}, P<.001)$ and interventricular septum/left ventricular posterior wall ratio 
Table 1 Demographic and echocardiographic characteristics of the patients

\begin{tabular}{|c|c|}
\hline Variable & Value \\
\hline Age (y) & $74.2 \pm 8.4$ \\
\hline Women & $28(48 \%)$ \\
\hline Weight (kg) & $73.9 \pm 15.2$ \\
\hline Height (cm) & $160.3 \pm 8.5$ \\
\hline Body surface area $\left(\mathrm{m}^{2}\right)$ & $1.77 \pm 0.19$ \\
\hline Atrial fibrillation & $11(19 \%)$ \\
\hline Interventricular septal thickness (mm) & $14.6 \pm 2.9$ \\
\hline LV posterior wall thickness (mm) & $12.9 \pm 2.1$ \\
\hline LV diastolic diameter (mm) & $44.5 \pm 8.3$ \\
\hline LV systolic diameter (mm) & $28.5 \pm 9.4$ \\
\hline LVEF (\%) & $58.7 \pm 14.8$ \\
\hline Left atrial volume index $\left(\mathrm{mL} / \mathrm{m}^{2}\right)$ & $39.5 \pm 18.9$ \\
\hline Peak gradient (mm Hg) & $69.8 \pm 27.1$ \\
\hline Mean gradient $(\mathrm{mm} \mathrm{Hg})$ & $42.1 \pm 17.3$ \\
\hline $\mathrm{SVi}\left(\mathrm{mL} / \mathrm{m}^{2}\right)$ & $38.1 \pm 13.2$ \\
\hline AVA, 2D TTE $\left(\mathrm{cm}^{2}\right)$ & $0.72 \pm 0.24$ \\
\hline AVAi, 2D TTE $\left(\mathrm{cm}^{2} / \mathrm{m}^{2}\right)$ & $0.41 \pm 0.12$ \\
\hline
\end{tabular}

$A V A i$, Aortic valve effective area index; $L V$, left ventricle; $L V E F$, left ventricular ejection fraction.

Data are expressed as mean \pm SD or number (percentage).

$(1.3 \pm 0.4$ vs $1.1 \pm 0.2, P<.01)$ and higher VTIs at the LVOT $(27.2 \pm 9.7$ vs. $20.2 \pm 4.5 \mathrm{~cm}, P<.05)$. CSA of the LVOT was more circular at the level of the HP of the aortic valve and more elliptical when measured at $8 \mathrm{~mm}$ proximal to the annular plane (Figure 3A). In fact, 30\% of patients showed circular shapes at the $\mathrm{HP}$, but only $3 \%$ did so at $8 \mathrm{~mm}$ (Figure $3 \mathrm{~B}$ ). LVOT anteroposterior diameter dimension was similar when measured by 3D TEE at any level. In contrast, LVOT lateral diameter increased from the HP of the aortic valve as measures were made more distal to the annulus $(23.1 \pm 3.3$ vs $24.9 \pm 3.3$ vs $27.3 \pm 3.9 \mathrm{~mm}, P<.001$; Table 2$)$.

\section{Two-Dimensional TTE: Annular Plane versus LVOT}

LVOT anteroposterior diameter was larger when measured at the HP than when measured more distal to the annular plane at the reference point $(20.8 \pm 2.3$ vs $19.8 \pm 2.3 \mathrm{~mm}, P<.01)$. Also, the values of the derived calculations were higher when diameter was measured at the HP: stroke volume index (SVi) $\left(41.2 \pm 14\right.$ vs $38.1 \pm 13.2 \mathrm{~mL} / \mathrm{m}^{2}$, $P<.01)$ and AVA $\left(0.79 \pm 0.29\right.$ vs $\left.0.72 \pm 0.24 \mathrm{~cm}^{2}, P<.001\right)$ (Table 2).

\section{D versus 3D Measurements}

The best correlation between 2D TTE and 3D TEE was at the HP level (ICC $=0.75 ; 95 \% \mathrm{CI}, 0.59$ to $0.86 ; P<.001$ ), whereas at the reference LVOT, the correlation with $3 \mathrm{D}$ TEE was very poor $(\mathrm{ICC}=0.35$; $95 \% \mathrm{CI},-0.21$ to $0.57 ; P=\mathrm{NS})$.

\section{Reclassification of AS Severity}

With TTE, when HP was selected, the number of patients with AVA $<1 \mathrm{~cm}^{2}$ was reduced from $50(86 \%)$ to $48(83 \%)(P<.01)$, and the number of patients with low flow $\left(\mathrm{SVi}<35 \mathrm{~mL} / \mathrm{m}^{2}\right)$ decreased from $24(41 \%)$ to $17(29 \%)(P<.001)$ (Figure 4$)$

\section{Reproducibility of the Measurements}

Intra- and interobserver reproducibility of the measurements is depicted in Table 3. The diameter measured by 2D TTE was more reproducible when measured at the HP of the aortic valve. The most reproducible measure was CSA by 3D TEE at the HP level (ICC $=0.96$; 95\% CI, 0.93-0.97). Intra- and interobserver variability was higher for lateral measurements than for anteroposterior ones.

\section{DISCUSSION}

\section{LVOT Morphology}

The most important finding of this study is the demonstration that in patients with degenerative AS, the LVOT is frequently funnel shaped, with an area that is reduced by $>15 \%$ in just $8 \mathrm{~mm}$. This funnel is more elliptical at the base and tends to be more circular at the valvular plane. Nonetheless, $70 \%$ of the patients in the study showed an elliptical morphology at the valvular plane, with a circularity index of $<0.9$. The reason for the elliptical morphology of the LVOT lies in the predominance of the medial-lateral diameter over the anteroposterior one. Moreover, the farther from the aortic annulus it is measured the medial-lateral diameter becomes larger, whereas the anteroposterior dimension does not change significantly, which results in a larger CSA (Figure 2). This funnel morphology, to the best of our knowledge, has not been previously described. Previous studies highlighting the elliptical morphology of the LVOT with multidetector computed tomography (MDCT) or $3 \mathrm{D} \mathrm{TEE}^{5-8}$ were restricted to the evaluation of the LVOT at only one plane, relatively closer to the aortic valve. In our study, with 3D TEE, we assessed the LVOT more extensively, performing three measurements in the $8 \mathrm{~mm}$ proximal to the aortic valve, and we have been able to demonstrate that this elliptical shape becomes more pronounced as it gets deeper into the left ventricle. The anteroposterior diameter evaluated with 3D TEE remains very similar at the three points of measurement, as opposed to the lateral diameter, which also increases as it gets deeper into the left ventricle (Figure 2). It should be taken into account that patients with severe hypertrophy of the interventricular septum may show a reduced CSA of the LVOT at 4 and $8 \mathrm{~mm}$ proximal to the annular plane. In our population, this inverted funnel shape was present in $18 \%$ of the patients, and it was associated with a proximal interventricular septal thickness $\geq 14 \mathrm{~mm}$ and an increased LVOT velocity (VTI $>25 \mathrm{~cm}$ ).

\section{D Transthoracic Echocardiographic Evaluation}

The continuity equation for AS evaluation was first proposed by Skjaerpe et al. ${ }^{13}$ in 1985 . In their article, the authors advocated for the measurement of LVOT diameter "just below the aortic valve." Current echocardiographic recommendations are not clear on which is the most adequate point for measuring the LVOT. Guidelines recommend measuring the LVOT diameter at 4 to $8 \mathrm{~mm}$ from the aortic valvular plane. ${ }^{4}$ Other authors propose measuring the LVOT at no more than $5 \mathrm{~mm}$ from the valvular plane, ${ }^{15}$ whereas others support measuring the LVOT just at the HP of the aortic valve.

In our study, LVOT dimensions on 2D TTE differed significantly on the basis of the point of measurement, whether the HP of the aortic valve or at 4 to $8 \mathrm{~mm}$ proximal to the valvular plane. Indeed, 


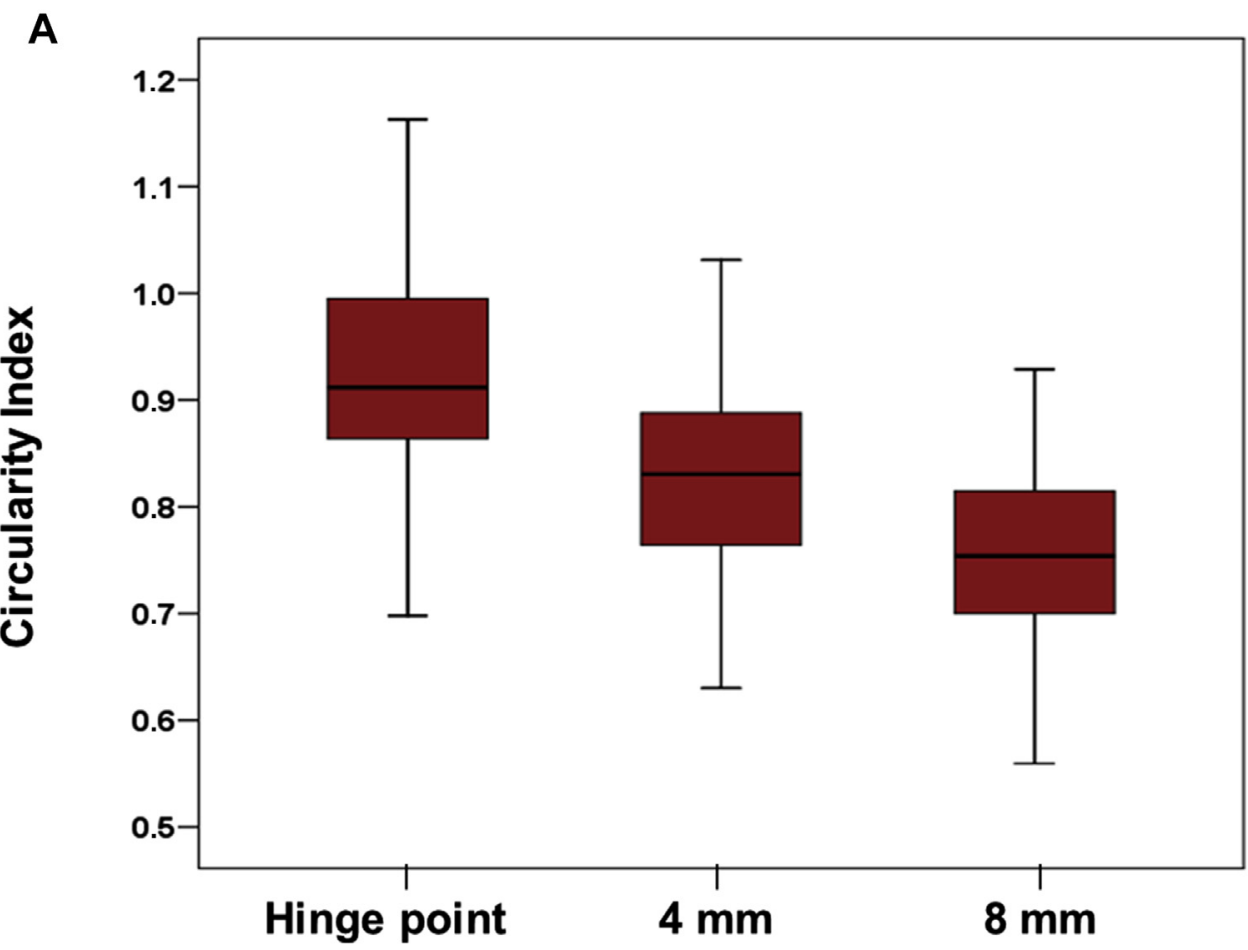

B

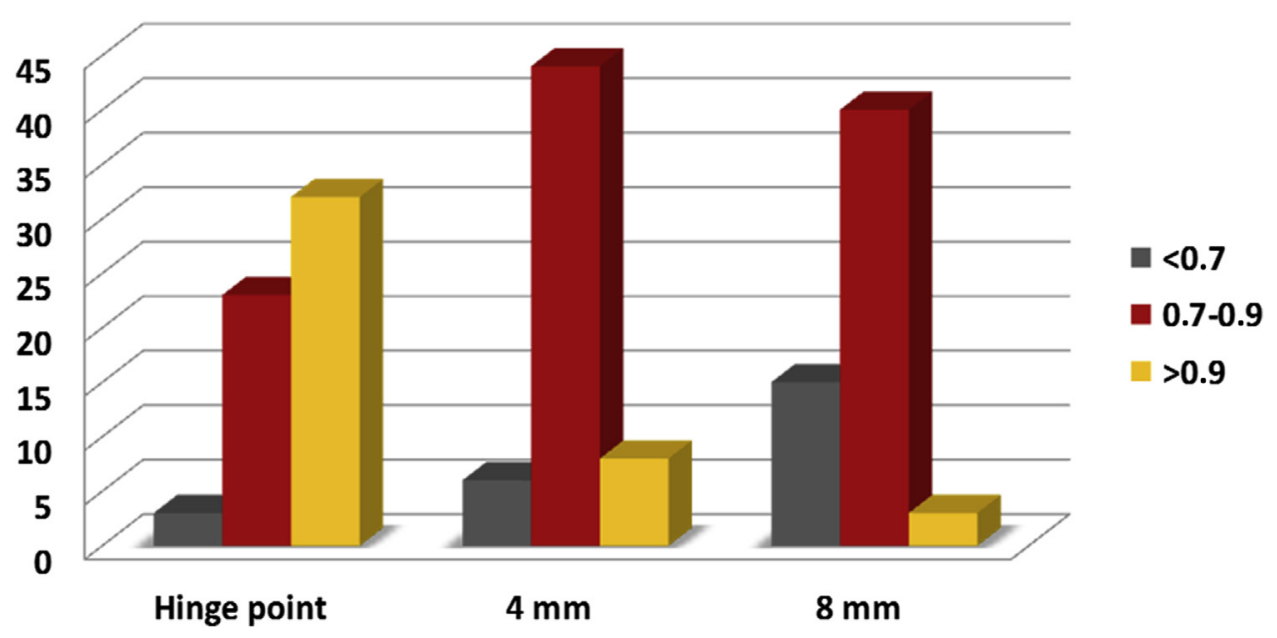

Figure 3 (A) Circularity index of the LVOT with 3D TEE at the three points of measurement. (B) Distribution of patterns of circularity index of the LVOT with 3D TEE at the three points of measurement.

measurement at the HP was significantly higher than measurement at 4 to $8 \mathrm{~mm}$ from the valvular plane and was very similar to that obtained with 3D TEE.

Choosing the best point to measure the anteroposterior diameter of the LVOT by 2D TTE is not easy. In our study, intra- and interobserver variability were acceptable and very similar at all points. Nevertheless, the farther from the aortic annulus it was measured, the smaller anteroposterior diameter of the LVOT was obtained by 2D TTE. When compared with 3D TEE, the best correlation was found for the measurement at the HP (Table 2). In this way, there were no significant differences between the two methods when the measurements were made at the HP. These results seem to support the initial description of the method of Skjaerpe et al. ${ }^{13}$ Furthermore, LaBounty et al. ${ }^{16}$ demonstrated in a previous study that effective orifice area calculated with the measurement of the LVOT at the annular diameter rather than the LVOT diameter 0.5 or $1 \mathrm{~cm}$ below the annulus resulted in the best agreement with AVA determined by invasive hemodynamics. In contrast, the use of subannular diameters resulted in a significant over- or underestimation of effective orifice area, depending on LVOT morphology (funnel 
Table 2 LVOT dimensions and derived calculations assessed by 2D TTE and 3D TEE at the HP of the aortic valve and at 4 to 8 mm of the annular plane

\begin{tabular}{|c|c|c|c|c|c|}
\hline \multirow[b]{2}{*}{ Variable } & \multicolumn{2}{|c|}{ 2D TTE } & \multicolumn{3}{|c|}{ 3D TEE } \\
\hline & LVOT & HP & HP & $4 \mathrm{~mm}$ & $8 \mathrm{~mm}$ \\
\hline APD $(\mathrm{mm})$ & $19.8 \pm 2.3^{*}$ & $20.8 \pm 2.3^{\dagger}$ & $20.8 \pm 2.7$ & $20.1 \pm 3.7^{\dagger}$ & $20.4 \pm 3.6^{\dagger}$ \\
\hline LD (mm) & - & - & $23.1 \pm 3.3$ & $24.9 \pm 3.3^{*}$ & $27.3 \pm 3.9^{\star}$ \\
\hline Cln & - & - & $0.91 \pm 0.09$ & $0.81 \pm 0.1^{*}$ & $0.75 \pm 0.09^{*}$ \\
\hline $\mathrm{CSA}\left(\mathrm{cm}^{2}\right)$ & $3.18 \pm 0.8^{*}$ & $3.44 \pm 0.8^{*}$ & $3.76 \pm 0.9$ & $4.05 \pm 1.1^{*}$ & $4.46 \pm 1.3^{\star}$ \\
\hline SVi $\left(m L / m^{2}\right)$ & $38.1 \pm 13.2^{*}$ & $41.2 \pm 14.1^{*}$ & $45.1 \pm 17.2$ & $47.8 \pm 15.9^{*}$ & $51.9 \pm 17.1^{*}$ \\
\hline $\operatorname{AVA}\left(\mathrm{cm}^{2}\right)$ & $0.72 \pm 0.24^{*}$ & $0.79 \pm 0.29^{\star}$ & $0.86 \pm 0.32$ & $0.92 \pm 0.32^{*}$ & $1.0 \pm 0.35^{*}$ \\
\hline AVA $<1 \mathrm{~cm}^{2}$ & $50(86 \%)^{*}$ & $48(83 \%)^{*}$ & 42 (72\%) & $34(58 \%)^{*}$ & $32(55 \%)^{*}$ \\
\hline $\mathrm{SVi}<35 \mathrm{ml} / \mathrm{m}^{2}$ & $24(41 \%)^{\star}$ & $17(29 \%)^{\star}$ & 15 (25\%) & $12(21 \%)^{\star}$ & $8(14 \%)^{\star}$ \\
\hline
\end{tabular}

$A P D$, Anteroposterior diameter; $C l n$, circularity index; $L D$, lateral diameter.

${ }^{*} P<.01$ compared with 3D transesophageal measurement at HP.

${ }^{\dagger}$ No significant difference.

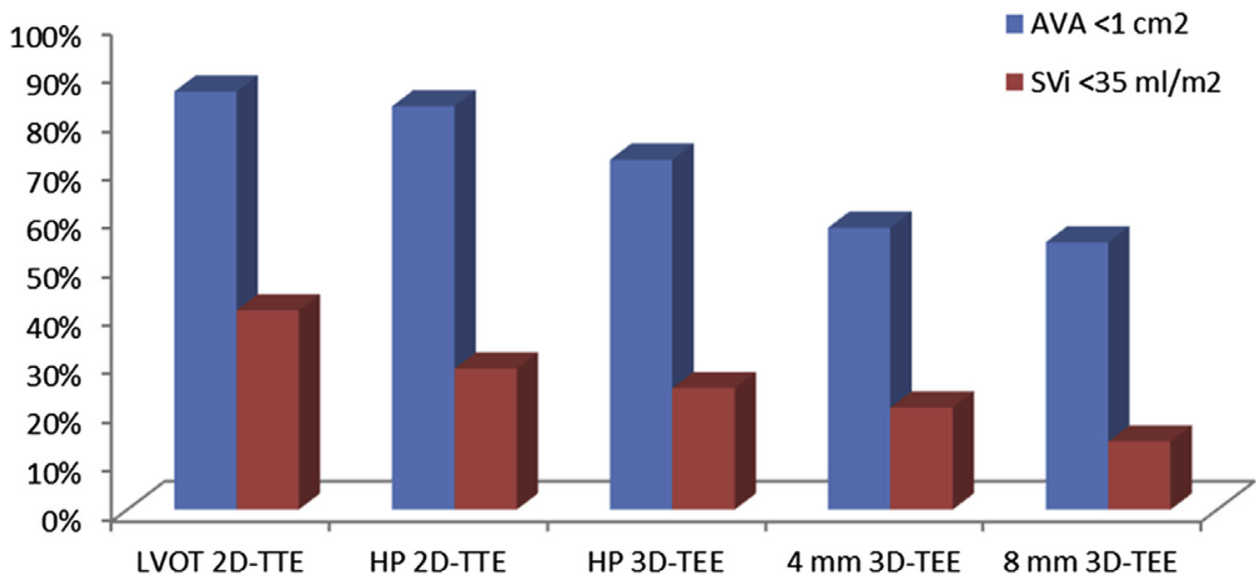

Figure 4 Proportion of patients with low-flow state $\left(\mathrm{SVi}<35 \mathrm{~mL} / \mathrm{m}^{2}\right)$ and severe AS $\left(A V A<1 \mathrm{~cm}^{2}\right)$ depending on the point of measurement of LVOT diameter with 2D TTE and 3D TEE.

shaped or hourglass shaped). This may contribute to the discrepancy with invasive measurement of AVA using the Gorlin equation.

\section{Clinical Implications}

The repercussions of the choice of the point of measurement are not negligible. In fact, in our study, when comparing the reference method of measuring the LVOT at 4 to $8 \mathrm{~mm}$ of the annular plane, with the measurement at the HP, the number of patients with severe AS (AVA $<1 \mathrm{~cm}^{2}$ ) decreased from 50 to 48 . Likewise, the number of patients with low-flow state $\left(\mathrm{SVi}<35 \mathrm{~mL} / \mathrm{m}^{2}\right)$ decreased from 24 to 17. This could partly explain the high variability in the prevalence of low-flow, low-gradient AS in the different series published. ${ }^{17-24}$ Although the reproducibility is similar in both LVOT measurements with 2D TTE, given the better correlation of LVOT diameter at the HP with 3D TEE, measurement of LVOT dimensions at the HP could be advocated as the reference approach.

On the other hand, according to the law of conservation of energy, the velocity of blood flow should increase in the same proportion as the area decreases along the LVOT. Therefore, the LVOT VTI will increase by $>15 \%$ as one moves distally in the LVOT toward the aortic valvular plane. This implies that an adequate evaluation of LVOT velocity should involve placing the sample volume as close as possible to the aortic valve plane, while being careful to avoid contamination of the echoes from the valve. ${ }^{4}$

\section{D Transesophageal Echocardiographic Methodology}

Three-dimensional TTE is an alternative method to measure the real dimensions of the LVOT. Previous studies using this method ${ }^{25,26}$ revealed the elliptical morphology of the LVOT and an underestimation of the calculation of AVA and SVi compared with the LVOT measured by 2D TTE. Tamborini et al. ${ }^{27}$ showed that 3D TTE is a reliable and valid imaging alternative to MDCT in preoperative transcatheter aortic valve implantation evaluation. Nevertheless, Tsang et al. $^{28}$ reported that in patients with calcified aortic valves, 3D TTE had the lowest accuracy with the largest variability compared with cardiac magnetic resonance and MDCT. Despite the good correlations, when comparing different imaging modalities, the lowest measurements are obtained with 2D TTE, followed by 3D TEE, and the highest measurements are obtained with MDCT. ${ }^{27,29-31}$ However, despite the accuracy of these new cardiac imaging 
Table 3 Intraobserver and interobserver reproducibility

\begin{tabular}{lll}
\hline LVOT dimension & $\begin{array}{c}\text { Intraobserver } \\
\text { ICC }(95 \% \mathrm{Cl})\end{array}$ & $\begin{array}{c}\text { Interobserver } \\
\text { ICC }(95 \% \mathrm{Cl})\end{array}$ \\
\hline 2D TTE & & \\
LVOT $(4-8 \mathrm{~mm})$ & $0.70(0.55-0.85)$ & $0.73(0.59-0.85)$ \\
LVOT (HP) & $0.75(0.57-0.86)$ & $0.72(0.55-0.81)$ \\
3D TEE & & \\
APD (HP) & $0.91(0.82-0.94)$ & $0.80(0.72-0.86)$ \\
APD $(4 \mathrm{~mm})$ & $0.81(0.74-0.88)$ & $0.80(0.71-0.89)$ \\
APD $(8 \mathrm{~mm})$ & $0.82(0.77-0.87)$ & $0.79(0.66-0.89)$ \\
LD (HP) & $0.73(0.55-0.84)$ & $0.61(0.39-0.79)$ \\
LD $(4 \mathrm{~mm})$ & $0.66(0.41-0.83)$ & $0.52(0.23-0.66)$ \\
LD $(8 \mathrm{~mm})$ & $0.68(0.42-0.82)$ & $0.53(0.31-0.69)$ \\
CSA (HP) & $0.96(0.93-0.97)$ & $0.91(0.85-0.92)$ \\
\hline CSA $(4 \mathrm{~mm})$ & $0.84(0.78-0.90)$ & $0.77(0.62-0.81)$ \\
CSA $(8 \mathrm{~mm})$ & $0.68(0.41-0.86)$ & $0.55(0.31-0.69)$ \\
\hline
\end{tabular}

$A P D$, Anteroposterior diameter; $L D$, lateral diameter.

techniques, it is important to acknowledge that the cutoff values to determine low flow and AVA should be reassessed, because the current cutoff values used for AS were only validated against clinical outcomes for standard 2D transthoracic evaluation.

Our methodology with 3D TEE and QLAB evaluation has been previously described by several groups. ${ }^{5-7,9,11,32,33}$ We obtained good reproducibility on anteroposterior diameters and CSA at $\mathrm{HP}$ and at $4 \mathrm{~mm}$ (ICC $>0.9$ ). The medial-lateral diameter and the measurements at $8 \mathrm{~mm}$ had lower but acceptable reproducibility $^{34}$ (ICC $=0.5-0.7$ ). The reason for these differences lies in the axial and lateral resolution of the technique. Our data on intraand interobserver variability are consistent with those previously reported. $^{5-7,9,11}$ Three-dimensional TEE provides more accurate information than 2D TEE, as it allows a cross-sectional plane at any level of the LVOT. ${ }^{32}$ The measurements with this technique have reproducibility comparable with that of MDCT, with reduced intraand interobserver variability. ${ }^{33,35}$ Because of this, the use of 3D TEE in aortic annular sizing for transcatheter aortic valve implantation has been recommended. ${ }^{33,36}$

\section{Limitations}

The main limitation of this study is the lack of a gold-standard imaging technique to establish comparisons. Despite the lack of available multidetector computed tomographic data with which to compare our echocardiographic results, previous studies have shown an excellent concordance in the elliptical shape obtained using both methods, with the magnitude of the dimensions the only variation identified. Indeed, LVOT dimensions were slightly larger when MDCT was used. Therefore, the LVOT morphology described in our study could be valid.

Whether our data, obtained in a rather elderly population, could be extrapolated to younger patients is unknown. Moreover, the sample size could be a limitation to the validity of the results regarding the comparison of LVOT measurements. Finally, the retrospective design of the study could limit somehow the interpretation of the results. This constraint was counteracted, however, by performing TTE and 3D TEE with a time interval of $<7$ days in the majority of patients and by doing both tests the same day for many of them.

\section{CONCLUSIONS}

Three-dimensional echocardiographic evaluation in patients with degenerative AS showed a funnel-shaped morphology of the LVOT in $>80 \%$ of patients. This funnel has elliptical bases, being more pronounced in the region of the LVOT farther from the annular plane. Its size and circularity differ significantly on the basis of the point of measurement. The assumption of a circular morphology cannot be accepted, as $>70 \%$ of patients have ellipse-shaped LVOTs at the HP of the aortic cusps. The best correlation between 2D TTE and 3D TEE is obtained when measured close to the annular plane. Our results support the recommendation of measuring the anteroposterior diameter of the LVOT close to the annular aortic plane to reduce measurement errors and AS misclassifications.

\section{REFERENCES}

1. Joint Task Force on the Management of Valvular Heart Disease of the Eu ropean Society of Cardiology (ESC), European Association for Cardio Thoracic Surgery (EACTS), Vahanian A, Alfieri O, Andreotti F, Antunes MJ, et al. Guidelines on the management of valvular heart disease (version 2012). Eur Heart J 2012;33:2451-96.

2. American College of Cardiology/American Heart Association Task Force on Practice Guidelines, Society of Cardiovascular Anesthesiologists, Society for Cardiovascular Angiography and Interventions, Society of Thoracic Surgeons, Bonow RO, Carabello BA, et al. ACC/AHA 2006 guidelines for the management of patients with valvular heart disease: a report of the American College of Cardiology/American Heart Association Task Force on Practice Guidelines (writing committee to revise the 1998 Guidelines for the Management of Patients With Valvular Heart Disease): developed in collaboration with the Society of Cardiovascular Anesthesiologists: endorsed by the Society for Cardiovascular Angiography and Interven tions and the Society of Thoracic Surgeons. Circulation 2006;114 e84-231.

3. Nishimura RA, Otto CM, Bonow RO, Carabello BA, Erwin JP, Guyton RA, et al. 2014 AHA/ACC guideline for the management of patients with valvular heart disease: executive summary: a report of the American College of Cardiology/American Heart Association Task Force on Practice Guidelines. J Am Coll Cardiol 2014;63:2438-88.

4. Baumgartner H, Hung J, Bermejo J, Chambers JB, Evangelista A, Griffin BP, et al. Echocardiographic assessment of valve stenosis: EAE/ASE recommendations for clinical practice. I Am Soc Echocardiogr 2009;22:1-23.

5. Saitoh T, Shiota M, Izumo M, Gurudevan SV, Tolstrup K, Siegel RJ, et al. Comparison of left ventricular outflow geometry and aortic valve area in patients with aortic stenosis by 2-dimensional versus 3-dimensional echocardiography. Am J Cardiol 2012;109:1626-31.

6. Saitoh T, Tanaka J, Furugen A, Harada K, Izumo M, Fukuoka Y, et al. Impact of energy loss index and valvuloarterial impedance in patients with aortic stenosis using three-dimensional echocardiography. Echocardiography 2015;32:654-9.

7. Ng AC, Delgado V, van der Kley F, Shanks M, van de Veire NR, Bertini M, et al. Comparison of aortic root dimensions and geometries before and af ter transcatheter aortic valve implantation by 2- and 3-dimensional trans esophageal echocardiography and multislice computed tomography. Circ Cardiovasc Imaging 2010;3:94-102.

8. Watanabe Y, Morice MC, Bouvier E, Leong T, Hayashida K, Lefèvre T et al. Automated 3-dimensional aortic annular assessment by multidetec tor computed tomography in transcatheter aortic valve implantation. JACC Cardiovasc Interv 2013;6:955-64.

9. Gaspar T, Adawi S, Sachner R, Asmer I, Ganaeem M, Rubinshtein R, et al Three-dimensional imaging of the left ventricular outflow tract: impact on aortic valve area estimation by the continuity equation. J Am Soc Echocardiogr 2012;25:749-57. 
10. Mor-Avi V, Sugeng L, Lang RM. Real-time 3-dimensional echocardiography: an integral component of the routine echocardiographic examination in adult patients? Circulation 2009;119:314-29.

11. Poh KK, Levine RA, Solis J, Shen L, Flaherty M, Kang YJ, et al. Assessing aortic valve area in aortic stenosis by continuity equation: a novel approach using real-time three-dimensional echocardiography. Eur Heart J 2008;29: 2526-35.

12. Lang RM, Badano LP, Mor-Avi V, Afilalo J, Armstrong A, Ernande L, et al. Recommendations for cardiac chamber quantification by echocardiography in adults: an update from the american society of echocardiography and the European Association of Cardiovascular Imaging. J Am Soc Echocardiogr 2015;28:1-39.

13. Skjaerpe T, Hegrenaes L, Hatle L. Noninvasive estimation of valve area in patients with aortic stenosis by Doppler ultrasound and two-dimensional echocardiography. Circulation 1985;72:810-8.

14. De la Morena G, Saura D, Oliva MJ, Soria F, González J, García M, et al. Real-time three-dimensional transoesophageal echocardiography in the assessment of aortic valve stenosis. Eur J Echocardiogr 2010;11:9-13.

15. Douglas PS, Waugh RA, Bloomfield G, Dunn G, Davis L, Hahn RT, et al. Implementation of echocardiography core laboratory best practices: a case study of the PARTNER I trial. I Am Soc Echocardiogr 2013;26:348-3583.

16. LaBounty TM, Miyasaka R, Chetcuti S, Grossman PM, Deeb GM, Patel HJ, et al. Annulus instead of LVOT diameter improves agreement between echocardiography effective orifice area and invasive aortic valve area. JACC Cardiovasc Imaging 2014;7:1065-6.

17. Pibarot P, Dumesnil JG. Low-flow, low-gradient aortic stenosis with normal and depressed left ventricular ejection fraction. J Am Coll Cardiol 2012; 60:1845-53.

18. Pibarot P, Dumesnil JG. Paradoxical low-flow, low-gradient aortic stenosis: new evidence, more questions. Circulation 2013;128:1729-32.

19. Lancellotti P, Magne J, Donal E, Davin L, O'Connor K, Rosca M, et al. Clinical outcome in asymptomatic severe aortic stenosis: insights from the new proposed aortic stenosis grading classification. J Am Coll Cardiol 2012;59: 235-43.

20. Hachicha Z, Dumesnil JG, Bogaty P, Pibarot P. Paradoxical low-flow, lowgradient severe aortic stenosis despite preserved ejection fraction is associated with higher afterload and reduced survival. Circulation 2007; 115: 2856-64.

21. Eleid MF, Sorajja P, Michelena HI, Malouf JF, Scott CG, Pellikka PA. Flowgradient patterns in severe aortic stenosis with preserved ejection fraction clinical characteristics and predictors of survival. Circulation 2013;128: 1781-9.

22. Adda J, Mielot C, Giorgi R, Cransac F, Zirphile X, Donal E, et al. Low-flow, low-gradient severe aortic stenosis despite normal ejection fraction is associated with severe left ventricular dysfunction as assessed by speckletracking echocardiography: a multicenter study. Circ Cardiovasc Imaging 2012;5:27-35

23. Mehrotra P, Jansen K, Flynn AW, Tan TC, Elmariah S, Picard MH, et al. Differential left ventricular remodelling and longitudinal function distinguishes low flow from normal-flow preserved ejection fraction low-gradient severe aortic stenosis. Eur Heart J 2013;34:1906-14.

24. González-Cánovas C, Muñoz-Esparza C, Oliva MJ, González-Carrillo J, López-Cuenca A, Saura D, et al. Severe aortic valve stenosis with lowgradient and preserved ejection fraction: a misclassification issue? Rev Esp Cardiol 2013;66:255-60

25. Doddamani S, Grushko MJ, Makaryus AN, Jain VR, Bello R, Friedman MA, et al. Demonstration of left ventricular outflow tract eccentricity by 64-slice multi-detector CT. Int J Cardiovasc Imaging 2009;25: 175-81.

26. Khaw AV, von Bardeleben RS, Strasser C, Mohr-Kahaly S, Blankenberg S, Espinola-Klein C, et al. Direct measurement of left ventricular outflow tract by transthoracic real-time 3D-echocardiography increases accuracy in assessment of aortic valve stenosis. Int J Cardiol 2009;136:64-71.

27. Tamborini G, Fusini L, Muratori M, Cefalù C, Gripari P, Ali SG, et al. Feasibility and accuracy of three-dimensional transthoracic echocardiography vs. multidetector computed tomography in the evaluation of aortic valve annulus in patient candidates to transcatheter aortic valve implantation. Eur Heart J Cardiovasc Imaging 2014;15:1316-23.

28. Tsang W, Bateman MG, Weinert L, Pellegrini G, Mor-Avi V, Sugeng L, et al. Accuracy of aortic annular measurements obtained from three dimensional echocardiography, CT and MRI: human in vitro and in vivo studies. Heart 2012;98:1146-52.

29. Jilaihawi H, Kashif M, Fontana G, Furugen A, Shiota T, Friede G, et al. Cross-sectional computed tomographic assessment improves accuracy of aortic annular sizing for transcatheter aortic valve replacement and reduces the incidence of paravalvular aortic regurgitation. J Am Coll Cardiol 2012;59:1275-86.

30. Pibarot P, Clavel MA. Left ventricular outflow tract geometry and dynamics in aortic stenosis: implications for the echocardiographic assessment of aortic valve area. J Am Soc Echocardiogr 2015;28:1267-9.

31. Mehrotra P, Flynn AW, Jansen K, Tan TC, Mak G, Julien HM, et al. Differential left ventricular outflow tract remodeling and dynamics in aortic ste nosis. J Am Soc Echocardiogr 2015;28:1259-66.

32. Jilaihawi H, Doctor N, Kashif M, Chakravarty T, Rafique A, Makar M, et al. Aortic annular sizing for transcatheter aortic valve replacement using cross-sectional 3-dimensional transesophageal echocardiography. J Am Coll Cardiol 2013;61:908-16.

33. Hahn RT. Guidance of transcatheter aortic valve replacement by echocardiography. Curr Cardiol Rep 2014;16:442.

34. Fermanian J. Measurement of agreement between 2 judges. Qualitative cases. Rev Epidemiol Sante Publique 1984:32:140-7.

35. Khalique OK, Kodali SK, Paradis JM, Nazif TM, Williams MR, Einstein AJ, et al. Aortic annular sizing using a novel 3-dimensional echocardiographic method: use and comparison with cardiac computed tomography. Circ Cardiovasc Imaging 2014;7:155-63.

36. Zamorano JL, Badano LP, Bruce C, Chan KL, Gonçalves A, Hahn RT, et al. EAE/ASE recommendations for the use of echocardiography in new transcatheter interventions for valvular heart disease. Eur J Echocardiogr $2011 ; 12: 557-84$. 